\section{P2-542 POPULATION LEVEL INVESTIGATION OF HOSPITAL DENTAL ADMISSIONS FOR CHILDREN UNDER 5 YEARS WITH INTELLECTUAL DISABILITY}

doi:10.1136/jech.2011.142976m.69

${ }^{1} \mathrm{~L}$ Slack-Smith, ${ }^{*}{ }^{1} \mathrm{~L}$ Colvin, ${ }^{1} \mathrm{H}$ Leonard, ${ }^{3} \mathrm{~N}$ Kilpatrick, ${ }^{2} \mathrm{~L}$ B Messer. ${ }^{1}$ University of Western Australia, Perth, Western Australia, Australia; ${ }^{2}$ University of Melbourne, Melbourne, Victoria, Australia; ${ }^{3}$ University of Bristol, Bristol, UK

Introduction Population level detailed data describing hospital admissions for dental reasons in children with intellectual disability (ID) is lacking. This paper describes dental hospitalisations in children under five years of age identified with ID and compares with children without ID.

Methods Data were extracted from population databases in Western Australia linking midwives' data collected on all births with population data regarding birth defects, ID, hospitalisations and deaths. Children born 1983-1992 ( $\mathrm{n}=243031$; ID=3522) were studied as these were the birth years where ID data were available; dental hospitalisations $(\mathrm{DH})$ up to the fifth birthday were utilised.

Results Linked data were examined for those who had had a $\mathrm{DH}$ including 214 children with ID $(\mathrm{DH}=246)$ and for 6119 children without ID ( $\mathrm{DH}=6713)$. Children with ID were more likely to have a dental hospitalisation (OR 2.47; 95\% CI 2.14 to 2.84). For those with ID, having a dental hospitalisation was associated with factors including being privately insured (1.41; 1.06 to 1.87 ), having a birth defect $(2.24 ; 1.69$ to 2.96$)$ and living in an area without fluoridated water supply (2.09; 1.16 to 3.75$)$.

Conclusion Linkage of population databases can provide valuable information on dental hospitalisations for children with intellectual disability and assist in planning appropriate services.

\section{P2-543 MORTALITY FROM ROAD TRAFFIC INJURIES IN CAMEROON: THE NEGLECTED EPIDEMIC}

doi:10.1136/jech.2011.142976m.70

${ }^{1,4} \mathrm{~J}$ Sobngwi-Tambekou, ${ }^{*}{ }^{2} Z$ Ngoumbé, ${ }^{3} \mathrm{~A}$ Ngouyamsa, ${ }^{2} \mathrm{~S}$ Sidibé. ${ }^{1}$ Université Catholique d'Afrique Centrale, Yaoundé, Cameroon; ${ }^{2}$ Ministry of transport, Yaoundé, Cameroon; ${ }^{3}$ Ministry of defense, yaoundé, Cameroon; ${ }^{4}$ Recherche-Santé-Developpement, Yaoundé, Cameroon

Objective Developing countries are experiencing the highest burden of road traffic injuries (RTI) with more than $85 \%$ of road traffic deaths worldwide. Epidemiologic data are scarce in sub Saharan Africa to inform interventions. We aimed to estimate RTI-related mortality and assess the burden on vulnerable road users in Cameroon.

Methods We conducted a study of all police reports of road accidents involving personal injury for the years 2007-2009, over the entire $10275 \mathrm{~km}$ interurban road network of Cameroon, a 20-million inhabitants sub Saharan African country. We analysed crash type, type of road user, impact type, crash severity, number of casualties, and fatality. Full reports were available for $64.5 \%$ of all reported accidents. Results The average daily traffic was 159914 vehicles/year. A total of 2074 crashes were recorded in 2007, 2420 in 2008, and 2091 in 2009, causing 1257, 1433 and 1274 deaths in 2007, 2008, and 2009 respectively, and yielding a crude average of 128 fatalities per 100 million kilometres driven, 60 times higher than in Europe. The average mortality rate per road accident varied from 60.5 to $61.4 \%$ across the years. Pedestrians accounted for $30.3 \%$ of all deaths, and $34.2 \%$ of deaths resulted from crashes involving motorcycle users. Vulnerable road users, including pedestrians and motorcycle users represented $51 \%$ of all deaths.

Conclusion Road traffic injury is a major cause of death in Cameroon. Further research is warranted to develop context-appropriate and effective prevention strategies to reduce this epidemic and protect the particular at-risk road user groups.

\section{P2-544 INFANT MORTALITY ESTIMATION IN BRAZIL: RESULTS OF A PROACTIVE SEARCH OF DEATHS AND LIVE BIRTHS IN POOR REGIONS}

doi:10.1136/jech.2011.142976m.71

${ }^{1} \mathrm{C}$ Szwarcwald, ${ }^{* 1}$ O Morais. ${ }^{1}$ Fundação Oswaldo Cruz, Rio de Janeiro, Brazil; ${ }^{2}$ Ministry of Health, Brasilia, Brazil

Introduction In view of the limitations of indirect demographic techniques for infant mortality estimation in Brazil, the current strategy is to improve vital information. This paper presents results of a proactive search of deaths and live births in the Northeast and Amazonia.

Methods We analysed vital information from the mortality and live birth information systems. The adequacy analysis was based on five indicators calculated at the municipality level, per 3-year period, from 1996 to 2008. To complement secondary data analysis, in 2009, a proactive search was carried out in a sample of 133 municipalities in the Northeast and Amazonia, stratified by information adequacy, region and population size of the municipality of residence. Correction factors were calculated by strata and by Brazilian state.

Results Temporal trends of the adequacy indicators indicate advances in both information systems. In 2008, coverage of the mortality information system was $88 \%$ and coverage of infant deaths was $79 \%$. However, the proactive search of infant deaths showed large deficiencies of vital information in some poor areas, with only $52 \%$ infant deaths informed to the Mortality Information System. The correction factors varied from 1.11 to 1.62 , depending on the adequacy of vital information, and from 1.02 to 1.28 , by Brazilian state.

Conclusion The monitoring of vital events is an essential step in the process of reducing infant mortality. The analysis of local irregularities not only improves the quality of vital data registration, making possible to estimate the infant mortality rate directly, but also identifies priority areas for intervention.

\section{P2-545 HETEROGENEITY OF RISK FACTORS OF DENGUE VIRUS INFECTION IN AN URBAN SETTING}

doi:10.1136/jech.2011.142976m.72

${ }^{1} \mathrm{M}$ G Teixeira, ${ }^{*} \mathrm{M}$ L Barreto, ${ }^{1} \mathrm{M}$ D C N Costa, 'L D A Ferreira, ${ }^{1} \mathrm{~V}$ Morato, ${ }^{1} \mathrm{P}$ F Vasconcelos, ${ }^{2} \mathrm{~S}$ Cairncross. ${ }^{1}$ Federal University of Bahia, Salvador, Bahia, Brazil; ${ }^{2}$ London School of Hygiene and Tropical Medicine, London, UK; ${ }^{3}$ Evandro Chagas Institute, Belem, Para, Brazil

Introduction Although it has been established that poverty is one of the determinants of the majority of infectious and parasitic diseases, in the case of dengue this is still a matter of some controversy. The objective of this study was to describe the distribution of dengue seroprevalence and seroincidence and to investigate the relationship between the intensity of virus circulation and the population's living conditions or between group immunity and Aedes aegypti infestation rates, in different intra-urban spaces within a large city in north-eastern, Salvador-Brazil.

Methods A prospective study was conducted by means of serological investigations among a sample of people living in 30 different spaces ("sentinel areas") in the city of Salvador, which was selected according to extreme differences in living conditions.

Results High rates of seroprevalence $(67.7 \%)$ and seroincidence $(70.6 \%)$ were found for the circulating serotypes (DENV-1 and 2 ). 
The seroincidence was high (55\%) even when the group immunity had already been partially established (42\%) and the Ae. aegypti infestation rates were relatively low $(<3 \%)$. Contrary to the ecological analysis, at the individual level, substantial heterogeneity in dengue exposure was observed.

Conclusion The observation that the dengue virus in our environment does not respect social spaces strengthens the principle that vector control measures must always be universally applied in each territory. On the other hand, the identification of specific risk factors in the domestic domain may indicate a need for other evidencebased interventions which can help to eliminate the disease from cities such as Salvador.

\section{P2-546 AGE AT DIAGNOSIS AFFECTS ADHERENCE TO NATIONAL BREAST CANCER GUIDELINES REGARDING LOCAL THERAPY}

doi:10.1136/jech.2011.142976m.73

W van de Water, E Bastiaannet, ${ }^{*}$ A J M de Craen, C J H van de Velde, G J Liefers. Leiden University Medical Centre, Leiden, The Netherlands

Introduction In the Netherlands, breast cancer patients are treated according to national guidelines. Despite comprising a large proportion, elderly breast cancer patients are underrepresented in clinical trials. Therefore it is questionable whether guideline recommendations can be extrapolated from a young to a heterogeneous elderly population. Aim of this study was to assess age specific guideline adherence.

Methods Data were extracted from the Dutch Cancer Registration. Overall, 83982 patients without distant metastases, diagnosed between 2002 and 2008 were included. Adherence was assessed for breast surgery (BS), type of surgery (TS), axillary surgery (AS) and radiotherapy $(\mathrm{RT})$. Analyses were stratified by age $(<65,65-74$ and $\geq 75$ years)

Results Adherence decreased with increasing age (BS <65, 99\%; $65-74,98 \%$; $\geq 75,75 \%$; $<<0.001$. TS $69 \%$; 66\%; 42\% respectively, $\mathrm{p}<0.001$. AS 96\%; 94\%; 68\% respectively, $\mathrm{p}<0.001$. RT 91\%; 93\%; $83 \%$ respectively, $\mathrm{p}<0.001)$. BS and AS non adherence were explained by under treatment. For TS and RT, young patients were non adherent because of over- and under treatment. In the elderly, under treatment was the main reason for non adherence (under treatment TS 84\%; 91\%; 97\% respectively, p $<0.001$. RT 34\%; $50 \% ; 80 \%$ respectively, $\mathrm{p}<0.001)$. Over time adherence to breast surgery declined for elderly patients $(p<0.001)$. For other local therapies, the gap between young and elderly patients remained similar.

Conclusion These results emphasise the different approach and treatment of elderly breast cancer patients. Further age specific studies in breast cancer patients are necessary to investigate whether adherence to current guidelines results in optimal outcomes for elderly patients.

\section{P2-547 TILE: MUSCULOSKELETAL DISORDERS AND ASSOCIATED FACTORS AMONG NURSES IN SRI LANKA}

doi:10.1136/jech.2011.142976m.74

${ }^{1} \mathrm{~S}$ Warnakulasuriya, ${ }^{*} \mathrm{R}$ Peiris Jone, ${ }^{2} \mathrm{~A}$ R Wickramasinghe, ${ }^{3} \mathrm{~N}$ Sathiakumar. ${ }^{1}$ Faculty of Medical Sciences, University of Sri Jayewardenapura, Colombo, Sri Lanka; ${ }^{2}$ Faculty of Medicin, University of Kelaniya, Ragama, Sri Lanka; ${ }^{3}$ School of Public Health, University of Alabama, Berminham, USA

Introduction Work related musculoskeletal disorders (WMSDs) have significant impacts in labour intensive occupational groups. Lifting, moving, transferring and repositioning of patients and long hours spent standing during work makes nurses vulnerable to WMSDs.

Objective To determine the prevalence and risk factors associated with WMSDs among nurses.

Methods We randomly selected 237 nurses attached to National hospital of Sri Lanka and Colombo South Teaching hospitals, and interviewed them using a pre-tested questionnaire ascertaining WMSDs in the past year. Descriptive, $\chi^{2}$ tests and multivariate logistic regression analyses were performed.

Results Results indicated that $21 \%$ of nurses had at least one WMSD. Back pain was the most common WMSD occurring in $44 \%$ of nurses. The prevalence of other WMSDs included pain in the knee $(30 \%)$, neck $(19 \%)$, shoulder $(16 \%)$ wrist and arm $(13 \%)$ and elbow (4\%). Lower back pain was significantly associated with time pressure $(\mathrm{OR}=2.14,95 \% \mathrm{CL} 1.17$ to 3.88$)$, job dissatisfaction $(\mathrm{OR}=6.84,95 \%$ CL 1.46 to 31.94$)$, lifting more than $25 \mathrm{~kg}$ during a normal duty shift ( $\mathrm{OR}=2.16$, CI 1.26 to 3.7), and unhappiness during the past month $(\mathrm{OR}=2.74,95 \% \mathrm{CI} 1.06$ to 7.08$)$. Shoulder pain was significantly associated with job dissatisfaction $(\mathrm{OR}=4.15,95 \%$ CI 1.24 to 13.87$)$ and unhappiness ( $\mathrm{OR}=2.98,95 \%$ CI 1.11 to 7.98 )

Conclusion The present study shows that nurses have a high prevalence of MSDs. The predominant ergonomic problem is lower back pain, followed by pain in the knee, neck and shoulder areas. Interventional studies are needed to address WMSDs among nurses, particularly for lower back pain.

\section{P2-548 GERIATRIC MENTAL HEALTH AND RELIGION: RESULTS FROM THE KRAKOW STUDY ON THE RELATIONSHIP BETWEEN RELIGIOUS INVOLVEMENT AND HEALTH IN OLDER AGE}

doi:10.1136/jech.2011.142976m.75

B Wozniak, ${ }^{*}$ K Zawisza, B Tobiasz-Adamczyk. Jagiellonian University Medical College, Krakow, Poland

Background Taking into account the prevalence of religiosity in the population of Polish older people inclusion of religiosity measures in mental health research is needed. The aim of the study was to investigate the relationship between religious involvement and mental health in older age.

Methods The sample population consisted of 350 randomly selected people aged 65 and over, living in Krakow. Religiosity was measured by assessing the following dimensions: ritualistic, experiential, ideological, consequential and intellectual. Religious coping and support were also measured. Health was assessed using SF-20, HADS, Will-to-live, DUKE Social Support and Loneliness Scales. Statistical analysis was done using K-means cluster analysis and multivariate regression analysis.

Results Two clusters were extracted in cluster analysis: cluster 1 (those who scored higher on each dimension of religiosity, ie, more religiously involved) and cluster 2 (less involved). Multivariate linear regression analysis showed that those more involved had better mental health $(\beta=0.182)$, were less depressed $(\beta=-0.236)$ and had more will to live $(\beta=0.186)$ in comparison with those less involved. Regression analysis for particular dimensions of religiosity showed that consequential and experiential dimension significantly influenced the level of depression ( $\beta=-0.202$ and -0.185 respectively); consequential dimension also influenced mental health $(\beta=0.228)$. Respondents who were engaged in positive religious coping responses had better mental health than those who didn't $(\beta=0.240)$. Emotional support provided, received and anticipated from the members of religious congregation decreased the level of social loneliness ( $\beta=-0.229,-0.238$ and -0.313 respectively).

Conclusions Religious involvement is a significant predictor of mental health in older age. 